\title{
LA NIÑEZ Y ADOLESCENCIA VÍCTIMAS DE ABUSO SEXUAL, COMUNIDAD CAÑO EL SAPOTE, MUNICIPIO DE WASLALA, 2012
}

\author{
Noemí Jarey Blandón Rodríguez ${ }^{[1]}$ \\ Yerlis Margine Rodríguez Castillo ${ }^{[2]}$ \\ Angélica Leonor Ruíz ${ }^{[3]}$
}

\section{Resumen}

Se ha abordado la situación de la niñez y adolescencia víctimas de abuso sexual, realizada en la comunidad Caño el Sapote del municipio de Waslala, 2012. La metodología utilizada es cualitativa con enfoque descriptivo al abordarse casos y formas de abuso sexual en la niñez; además, se manifiestan las consecuencias que asumen cada niña, niño y adolescente sobreviviente de abuso sexual. También se ha descrito la ruta de acceso a la justicia. Las técnicas utilizadas fueron entrevistas semi- estructurada, grupo focal y revisión documental.

Los resultados demuestran que el abuso sexual es una de las formas de violencias que se viven dentro de las familias y personas cercanas a ella en todos los ámbitos y espacios sociales. Los resultados reflejan desconocimientos de lo que es en sí el abuso sexual por parte de la mayoría de las personas, acontecimiento que sucede dentro de la sociedad, pero por una u otra razón no se rompe el silencio, no existe igualdad por la posición en la pirámide social.

Palabras clave: abuso sexual; consecuencias, desconocimientos, nivel social, violencia.

\section{Summary}

The present study addresses the situation of children and adolescents victims of sexual abuse, in the community of Caño El Sapote, municipality of Waslala, 2012. The methodology that was used is qualitative with a descriptive approach when addressing cases and forms of sexual abuse in children. We make use of techniques based on semi- structured interviews, focus groups and document review. The research also manifests the consequences that each girl, boy and adolescent survivors of sexual abuse assumes. As well, it describes the path to justice.

\footnotetext{
[1] Licenciada en Sociología con mención en Autonomía. Docente de Sociología.

[2] Licenciada en Sociología con mención en Autonomía. Docente de Sociología

[3] Máster en Antropología social. Secretaria Académica del Recinto Las Minas. leoruiz_71@hotmail.com
} 
The results show that sexual abuse is one of the forms of violence that are experienced within families and people close to them in all areas and social spaces. The results reflect that most people are lacking information of what is sexual abuse, an event that occurs in society and has become very common, but for whatever reason the silence does not break, and there is no equality due to the position in the social pyramid.

Keywords: Sexual abuse, consequences, unfamiliar, social position.

\section{Introducción}

El abuso sexual en la niñez y la adolescencia es un problema no sólo personal y familiar, sino que también de índole social al atentar contra la salud pública. Hablar de abuso sexual es referirse al adueñarse de manera violenta de la vida de niños, niñas y adolescentes, alterando su normal desarrollo a partir del uso de su cuerpo y del abuso de su situación de indefensión o vulnerabilidad.

El tema es de gran importancia al poder alertar a los padres, madres y profesores sobre la situación de abuso que se está viviendo en el municipio, y así también crear consciencia en todas las instituciones para que resuelvan los casos que se presentan para brindarles a las víctimas una razón de vida por la cual hay que seguir adelante.

En este estudio se caracterizan los casos y formas de abuso sexual en la niñez y adolescencia, se describen los factores que inciden y las consecuencias en el abuso sexual, se explica la ruta de acceso a la justicia.

\section{Revisión de Literatura}

\section{Casos y formas de abuso sexual en la niñez y adolescencia}

El abuso sexual es un patrón de comportamiento caracterizado por el abuso de poder, la intimidación y la coerción. Es un tema común en toda la sociedad que no distingue ningún estatus social, un fenómeno mundial en el que los más violentados son los niños y niñas (Plank A, 2005, p.76, Castillo, Amador, 2006).

La mayoría de los casos presentan las mismas condiciones del entorno, en su mayoría son familias de escasos recursos económicos y sobre todo un bajo nivel educativo, son desprotegidos, carecen del afecto y cariño tanto de sus madres y padres quienes los obligan a realizar todo tipo de trabajo (Juárez, Montenegro, 2007, p.21).

El abuso sexual infantil se da en la intimidad del hogar, la mayor parte de las veces es por el padre, tío, padrastro u otro mayor a la víctima, y que goza de la absoluta 
confianza de la familia, aunque también se registran pocos casos de mujeres abusadoras (Arauz, Matamoros, S/f, p.4).

Es importante mencionar las diferencias cruciales entre la violación que sufre una persona adulta y el abuso sexual que han sufrido siendo niñas y niños. En la mayoría de los casos de violación a una persona adulta el violador huye después de cometer su delito y deja a su víctima abandonada; caso contrario el abusador vive, con frecuencia en la misma casa de su víctima y se presenta ante ella, por la posición que ocupa en la familia (Norori L, 2009, p.8).

Dentro de este tema se presentan diversas formas de abuso sexual que a continuación se mencionara:

- Exposición: Es mostrar los órganos sexuales de una manera inapropiada, como en el exhibicionismo (MCAS, 2007, p. 6).

- Voyeurismo: Impulsos o comportamientos sexuales que implican el hecho de observar ocultamente a niñas, niños o adolescentes desnudándose o sin ropa (Save the Children, 2005., p. 8).

- Caricias: Esta se da por tocamientos o caricias en los genitales de otro, incluyendo la masturbación para cualquier contacto sexual, menos la penetración.

- Violación: Penetración en la vagina, ano o boca con cualquier objeto sin el consentimiento de la persona.

- Penetración digital: Inserción de un dedo en la vagina o en el ano.

- Coito vaginal o anal: Contacto genital oral.

- Lenguaje sexualizado: Es el uso de palabras inapropiadas, propuestas de tipo sexual erotizado.

- Filmar cuerpos desnudos de los menores.

- Penetración anal o vaginal con un objeto.

\section{Factores que inciden en el abuso sexual en menores de edad}

Los factores que inciden en el abuso sexual en la niñez y adolescencia están relacionados a las condiciones que favorecen el uso de la violencia intrafamiliar, asociada a un mayor riesgo de sufrir abusos sexuales por las condiciones de: pobreza, bajo nivel cultural, viviendas inadecuadas, abuso del alcohol. Situaciones familiares como: ausencia de los padres biológicos, incapacidad o enfermedad de la madre o tutores.

Los conflictos entre los padres (indiferencia, enfados, peleas, separaciones y divorcios). El creer que es tarea de la madre y no del padre el cuido y protección de los menores. Las relaciones pobres, escasas o deficitarias por algún motivo con los padres. El hecho de tener padrastro o madrastra (Fundación PLAN, S/f). 
El abuso sexual en la niñez se da por el abuso de poder. En el abuso sexual infantil, la autoridad y el poder están a favor del abusador, en la sociedad que vivimos hay tanta desigualdad, en la que todavía se le da socialmente mayor valor a un hombre que a una mujer, tienen mayor valor las personas adultas que los niños y las niñas a las que se les exige respeto y obediencia; pero tal problema no es el poder, sino en el uso que se hace de él; las relaciones que se establecen a partir de este pueden ser de abuso o de respeto (Arauz, Matamoros, Ibíd. p.4).

\section{Consecuencias del abuso sexual en niñas, niños y adolescentes}

Las secuelas o consecuencias del abuso sexual en un niño son muchas y pueden servir como indicadores de tal situación. Las repercusiones pueden ser a corto o largo plazo y son manifestaciones clínicas inespecíficas, difíciles de identificar como emergentes del abuso sexual, expuestas entre las cuales mencionamos las siguientes: (Aveiga, Escandon, S/f).

A largo plazo tenemos

\section{Físicas:}

Dolores crónicos generales, hipocondría o trastornos psicosomáticos, alteraciones del sueño y pesadillas constantes, problemas gastrointestinales y desorden alimentario.

\section{Conductuales:}

Intento de suicidio, consumo de drogas y alcohol y trastorno de identidad.

\section{Emocionales:}

Depresión, ansiedad, baja autoestima y dificultad para expresar sentimientos.

\section{Sexuales:}

Fobias sexuales, disfunciones sexuales, falta de satisfacción o incapacidad para el orgasmo, alteraciones de la motivación sexual, mayor probabilidad de sufrir violaciones, entrar en la prostitución y dificultad para establecer relaciones sexuales.

\section{Sociales:}

Problemas de relación interpersonal, aislamiento y dificultades de vinculación afectiva con los hijos. 
A largo plazo, aunque los efectos son comparativamente menos frecuentes que a corto plazo, el trauma no sólo no se resuelve sino que suele transitar de una sintomatología a otra. Con todo, no es posible señalar un síndrome característico de los adultos que fueron objeto de abusos sexuales en la infancia o adolescencia.

Existen numerosos condicionantes de la pervivencia de efectos a largo plazo, como puede ser, entre otros, la existencia en el momento de los abusos de otro tipo de problemas en la vida del niño (maltratos, divorcio de los padres, etc.) e, incluso, en muchos casos los efectos aparecen provocados por circunstancias negativas en la vida adulta (problemas de pareja, en el trabajo (Echebúrua y Guerricaechevarria, 2005).

Los indicios de que hay un abuso sexual son las siguientes, las cuales se dan a corto plazo en los menores.

\section{Físicas:}

Pesadillas y problemas de sueño, cambios de hábitos de comida y pérdida de control de esfínteres.

\section{Conductuales:}

Consumo de drogas y alcohol, fugas, conductas auto lesivas o suicidas, hiperactividad, bajo rendimiento académico.

\section{Emocionales:}

Miedo generalizado, agresividad, culpa y vergüenza, ansiedad, depresión, baja autoestima y rechazo al propio cuerpo.

\section{Sexuales:}

Conocimiento sexual precoz e impropio a su edad, masturbación compulsiva, exhibicionismo y problemas de identidad sexual.

\section{Sociales:}

Aislamiento, déficit en habilidades sociales, retraimiento social y conductas antisociales (Aveiga L. y Escandón C. Op. Cit. s.f).

\section{Ruta de acceso a la justicia}

Se define acceso a la justicia como el derecho de toda persona de acudir al sistema de justicia y obtener una adecuada respuesta. Es deber del Estado brindar un servicio 
público que cumpla con los principios rectores de: continuidad, adaptabilidad del servicio, igualdad, celeridad, gratuidad (Centeno, Matamóros, Pérez, 2009, P.9).

Como estrategia para garantizar el acceso a la justicia, las organizaciones han transitado por diferentes modelos, desde servicios legales alternativos para usuarias sin recursos económicos hasta acciones de incidencia política, y de negociación con el Estado para lograr una legislación y cambios a favor de los niños, niñas y adolescentes, pasando por acciones de interés público y la creación de las Comisarías de la Mujer la Niñez y la adolescencia (Centeno, Matamoros, Pérez. 2009. P.9).

\section{Materiales y métodos}

Esta investigación de tema Situación en la niñez y adolescencia víctimas de abuso sexual se llevo a cabo en la comunidad Caño El Sapote, municipio de Waslala, 2012.

Por el nivel de profundidad es de carácter descriptivo con un enfoque cualitativo, cuya población seleccionada son niños, niñas y adolescentes que han sufrido abuso sexual.

En el procesamiento de la información se entablaron conversaciones de costumbres familiares y ancestrales, momento para realizar preguntas que nos dieran salida al objetivo de la investigación, reunión con los personajes involucrados en el estudio donde se aplicó un grupo focal. La información obtenida se analizó por descriptor y fuente, para clasificarla de acuerdo a los instrumentos utilizados.

\section{Resultados y discusión}

\section{Casos y formas de abuso sexual en la niñez y adolescencia}

Las formas del abuso sexual más frecuente en la niñez y adolescencia se dan por tocamientos, caricias, besos hacia el menor por parte del abusador, en ocasiones los golpean y los obligan a que les acaricien los órganos genitales, penetración o violación, además por la observación, frases bonitas y gestos mal intencionados hacia las niñas y niños.

Esto último lo comento una madre de familia quien lo vivió en su adolescencia por parte de un tío, nos dice:

que esa forma de mirarla asediando su cuerpo e inocencia me estremeció, me incomodó, sentí terror y al mismo tiempo desprecio por este hombre, desprecio que ha perdurado a lo largo del tiempo, nos dice, yo prefiero no verlo porque me repugna su presencia. 
Al realizar comparación con la información encontrada, en la que relata (Norori L, 2009, P.8) se presentan diversas formas de abuso sexual tales como exposición, Voyeurismo, caricias, violación (penetración en la vagina, ano o boca con cualquier objeto sin el consentimiento de la persona), lenguaje sexualizado, filmar cuerpos desnudos de los menores.

Las niñas son mas abusadas sexualmente porque son engañadas con regalos, caramelos y también porque el poder lo tienen los hombres y son el sexo opuesto, son controladas fácilmente a través del poder ejercido por el hombre desde la familia, la escuela y la sociedad.

Tal situación los niños lo expresan así:

Corren más riesgo las niñas que los niños, son mas perseguidas por los maleantes, la niña no tiene la capacidad que tiene el varón son más débiles, el varón corre y busca como defenderse y que entre hombres no se pueden violar, porque estarían violando a su propio género.

Según lo expresado por Reyes, Vega y Weinberg, (2004). El abuso sexual es tan frecuente en los niños como en las niñas, situación que se vive a diario, con la diferencia que el abuso sexual en los niños es casi invisible, aunque esté sucediendo en la sociedad, debido al sistema patriarcal que radica en las familias desde las primeras generaciones y sustentado por tradiciones, costumbres, creencias religiosas y la misma cultura machista predominante en nuestra sociedad. Los abusos sexuales en infantes, no son un problema que afecte sólo al sexo femenino, sino también los niños sufren estas agresiones.

\section{Factores que inciden en el abuso sexual en menores de edad}

El abuso sexual en la niñez y adolescencia presenta una variedad de factores los cuales inciden de manera directa sobre ellos: Familiares, económicos, religiosos, sociales, jurídico.

Familiar: El abuso de poder, el machismo, por el simple hecho de ser hombre y creen que el dominio lo ejercen con el pene, debido a que no tienen dominio de su cuerpo dejándose llevar por los instintos y si le sumamos el adultismo. Salió a relucir mucho entre los entrevistados la frase "Hombre cabeza del hogar", la cual es un factor determinante para la violencia y el abuso dentro de las familias.

Económico: El factor económico es uno de los principales que incide de forma tan recurrente en el abuso sexual en la niñez. 
Religioso: La religión influye porque no les gusta que le hablen sobre tema de sexualidad y por consiguiente del abuso sexual, de esta manera se crea una barrera porque los niños deben de saber acerca de sexualidad y reproducción; pero en realidad es la manera en que son interpretados los diferentes términos y usados para hablar de este.

Social: dentro de los factores sociales son uno de los principales puntos de desencuentro para la niñez y adolescencia víctimas de abuso sexual porque es ahí en donde son duramente juzgados, agredidos, menospreciados de la manera más hostil que podamos imaginar. ¿Porque decimos esto? Porque estamos en una sociedad que ve el problema del otro como algo que no le compete, es problema de ellos y no mío. Ayudarlo sería como atraer un problema sobre tus hombros, y no tiene porque ser así. La falsa educación sexual, temas tabúes en todos los ámbitos sociales y por ende familiar.

En un estudio realizado por la UNICEF en el 2007 citado por Save the Children en Nicaragua de Causas y consecuencias del abuso sexual en los hogares, se destacó que uno de los factores que inciden en el abuso sexual en la niñez y adolescencia es la cultura, principalmente vivida como una costumbre ancestral con arraigo en el campo.

Jurídico: La justicia no actúa como debe ante estos hechos. La impunidad de casos se convierte en un factor determinante y que alienta, para que los abusadores se sientan con poder y derechos de seguir reincidiendo en los delitos. No hay instituciones que trabajen específicamente en esta problemática, si sabemos que muchas son las que trabajan en función de esto, pero son tantas las funciones que tienen que no hay un trabajo efectivo para la protección de los menores.

\section{Consecuencias del abuso sexual en la niñez y adolescencia}

En la aplicación de entrevistas sobre el abuso sexual en la niñez y adolescencia, a padres de familia, docentes, y representantes de organizaciones coinciden en que las consecuencias que enfrenta una víctima de abuso sexual son diversas como las que fueron evidentes durante la investigación de campo.

La niñez suele sufrir daños físicos y psicológicos a corto y largo plazo, que afectan su capacidad de aprender y de relacionarse socialmente, siente rechazo por el victimario, baja autoestima, el suicidio, el miedo, el llorar sin razón, los cambios de comportamiento, bajo rendimiento escolar, le cuesta dormir, la desintegración familiar.

Estos resultados están estrechamente relacionadas con lo que nos dice Echebúrua y Guerricaechevarria (2005. p.87), en el que este hecho constituye una experiencia traumática y es vivido por la víctima como un atentado contra su integridad física y psicológica, y no tanto contra su sexo, por lo que constituye una forma más de 
victimización en la infancia, con secuelas parcialmente similares a las generadas en los casos de maltrato físico, abandono emocional.

Todas estas consecuencias representan una profunda alteración en la dignidad e integridad de la niñez y adolescencia, y en muchas ocasiones se ponen en riesgo las vidas alterando así la armonía familiar dejando daños sobre su integridad física, emocional, daña la integridad del ser humano; no existe un calificativo que describa este hecho tan abominable. Ya que solamente la persona que lo vive lo puede expresar y a veces su manera de demostrarlo es con llanto.

\section{Ruta crítica de acceso a la justicia en la niñez y adolescencia víctimas de abuso sexual}

En las entrevistas aplicadas a líderes comunitarios el 80\% de estos, refiere que no se le da seguimiento a los casos presentados ante las autoridades y que por ende no brindan la atención adecuada para las víctimas; además de esto, las distancias que se recorren para llegar desde la comunidad hasta las autoridades correspondientes son muy extensas lo que dificulta la continuidad del proceso.

En la ruta de acceso a la justicia las víctimas que deciden romper el silencio y en algunos casos apoyados por su familia y líderes comunitarios se presentan a la Comisaría de la Mujer y la Niñez del municipio quienes registran la denuncia y posteriormente los envían al centro de salud para hacer el dictamen médico forense quien las remite nuevamente a la Comisaría de la Mujer y la Niñez, para dar inicio a la valoración y terapia psicológica, a quienes dependiendo la gravedad del caso se remite ya sea al juzgado local o de distrito para procesar al victimario.

Es muy importante mencionar que en el municipio no se cuenta con los recursos humanos en el órgano de la fiscalía, esto por la situación tan problemática de jurisdicción que atraviesa Waslala desde siempre y por ende los pobladores.

A lo cual expresa (Save the Children., 2005., p.9) que se debe seguir una guía en el proceso de atención a las víctimas, diez puntos fundamentales mencionados a continuación:

1. Escuchemos a las niñas y los niños.

2. Justicia para la niñez.

3. Responsabilidad compartida en la prevención del abuso sexual infantil: enfoque multisectorial y multidisciplinario.

4. Cómo dar cabida a la niñez en nuestros corazones: apropiación de la comunidad y el reto de los adultos. 
5. Las causas fundamentales del abuso sexual: sexualidad, poder y cultura.

6. Responsabilizar al abusador.

7. Participación y educación de las niñas y los niños en un entorno amigable para la niñez.

8. Una amenaza mortal: el SIDA.

9. Cómo promover nuestro mensaje mediante la incidencia.

10. Aprendizaje del proceso: planificación, seguimiento, evaluación y elementos de calidad.

\section{Conclusiones}

Las formas de abuso sexual en la niñez y adolescencia se dan por tocamientos, caricias, besos hacia el menor por parte del abusador, en ocasiones los golpean y los obligan a que les acaricien los órganos genitales, penetración o violación, además por la observación, frases bonitas y gestos malintencionados hacia las niñas y niños.

Los principales factores son familiares por ejemplo: el abuso de poder, la falsa educación sexual, la confianza que se les brinda a los demás, no escuchar a los niños y niñas, desintegración familiar y la migración, el hacinamiento. Resumiendo todo en tres palabras: sexualidad, poder y cultura. Sin obviar el económico, religioso, social y jurídico.

Las consecuencias del abuso sexual a la niñez y adolescencia son múltiples y se representa por variadas y devastadoras conductas en los inocentes y van desde la pérdida de la autoestima, irritabilidad, ausentismo social, estas y otras son secuelas de por vida para los abusados.

En este escenario las instituciones del Estado y la sociedad civil han venido desarrollando programas, proyectos y acciones para enfrentar este problema. Sin embargo, la real y efectiva implementación de la protección especial, aún constituye un reto y un compromiso que el Estado debe asumir conjuntamente con la sociedad civil y la participación de la comunidad y la familia. Pero no basta sólo con la creación de leyes y el diseño de estrategias, es darle seguimiento al cumplimiento de esos instrumentos, aspecto del que se carece en el país. 


\section{CIENCIAS SOCIALES}

\section{Lista de referencia}

Aráuz, Matamoros. (S/f.). Abuso Sexual de Niñas y Niños. Folleto Informatico. Matagalpa, Nicaragua, Grupo Venancia.

Aveiga L. Escandón C. (S/f.). Causas del Abuso Sexual Infantil y Herramientas para su detección y para la respuesta inicial y la protección de los derechos de los niños, niñas y Adolescentes frente a la violencia Sexual. Recuperado el o6 de 07 de 2012, de http://www.search.ask.com/web.

Castillo M. Amador M. (2006). Abuso Sexual Infantil Incidencias y Características. Recuperado el o6 de 07 de 2012, de http://cpanel.mcas.org.ni/mcascpanel/ userfiles/file/o6_abuso_sexual_infantil_incidencia_caracteristicas.PDF .

Centeno E, Matamoros S, Perez L. (2009). Recursos y Obstáculos en el acceso a la Justicia para mujeres víctimas de violencia. Matagalpa-Nicaragua, p.59.

Echebúrua E. Guerricaechevarria C. (2005). Abuso Sexual en la Infancia víctimas y agresores un enfoque clínico. Recuperado el o3 de o3 de 2012 de https://es.wikipedia. org/wiki/Abuso_sexual_infantil.

Fundación Plan (s.f.), Prevención del abuso sexual infantil. Recuperado el o2 de o8 de 2013, de html. plan.org.com/../ inicio/nuestro trabajo/nuestra compañía/ ponle 6 sentidos .

Juárez M. y Montenegro T. (2007). Cuando te roban la Vida. La Boletina, pp.36.

Movimiento Contra Abuso Sexual. (2007). Todas las Voces y Todas las Voluntades contra el Abuso Sexual. Managua-Nicaragua. MCAS.

Norori, L. (2009). Hablemos de Abuso Sexual. Nicaragua.

Plank A. (2005). Guía de Salud Sexual y Reproductiva para Adolescentes y Jóvenes. MadridEspaña: Horizont-300o, pp. 91.

Reyes, Vega y Weinberg. (2004). Necesitamos Poder Hablar pistas para prevenir y Apoyar. Edit. Puntos de Encuentro. La Boletina, pp. 50.

Save the Children. (2005). Informes sobre Abuso Sexual contra niños, niñas y adolescentes, Centro de Investigaciones en demiografía y salud. Nicaragua. CIDS- UNAN. pp 42.

MIFAMILIA/UNICEF. (2007). Diagnóstico de Causas y Manifestaciones del abuso sexual contra niños Niñas y Adolescentes. Nicaragua: UNICEF. 